\title{
La Société Française de Physique et les jeunes
}

La Société Française de Physique (SFP) vise à regrouper la communauté des physiciens et physiciennes dans un esprit collaboratif de bénévolat intergénérationnel, sans hiérarchie ni but lucratif. Les jeunes y ont toute leur place et si la SFP peut leur donner beaucoup, ils peuvent aussi enrichir cette dernière par leurs idées nouvelles, leur savoir-faire, leurs initiatives et leur énergie.

\section{Les événements organisés par les jeunes}

De nombreuses actions sont portées par les jeunes au sein de la SFP, notamment via la Commission Jeunes, forte d'une trentaine de membres actifs.

\section{French Physicists' Tournament}

Le French Physicists' Tournament (FPT) est organisé par la commission depuis 2014, avec le soutien de I'Académie des sciences et de plusieurs autres partenaires (CNRS, CEA, éditeurs, etc.). II connait une forte croissance et rassemble désormais tous les ans plus d'une cinquantaine d'étudiants de niveaux licence et master. Durant cinq mois, les étudiants travaillent en équipes avec leurs enseignants autour de problèmes expérimentaux ouverts; puis les différentes équipes se rassemblent durant deux jours pour présenter, en anglais, leurs résultats, critiquer et discuter de façon constructive les solutions des autres équipes. Le tournoi est une véritable formation à la recherche, et sa préparation est valorisée en crédits ECTS par tous les établissements qui le préparent. De plus, une revue internationale est en cours de lancement avec EDP Sciences pour permettre aux étudiants de publier leurs travaux (en lien avec le tournoi ou non). Enfin l'équipe gagnante représente la France lors de l'International Physicists' Tournament (IPT), cette année du 8 au 13 avril à Göteborg, en Suède !

\section{Les Rencontres des Jeunes Physicie ${ }^{\mathbf{n}} \mathbf{n}{ }^{\mathbf{s}}$}

Les Rencontres des Jeunes Physicie ${ }^{n} n^{e_{s}}$ (RJP), créées en 2013 par la section Paris-Centre, rassemblent tous les ans durant une journée environ 200 doctorants et postdoctorants de la région parisienne issus de tous les domaines de la physique (voir l'article sur les RJP 2016, p. 40). Le but est de présenter, de façon aussi pédagogique que possible, un large panorama des thématiques actuelles à travers les présentations des participants. C'est aussi l'occasion d'échanger entre jeunes chercheurs dans un cadre moins formel que celui des conférences habituelles, tout en conservant une exigence scientifique forte. Ce sont des lieux de discussions et d'échanges uniques en leur genre. Dans I'optique de développer ces rencontres dans d'autres villes, nous avons rédigé un court document d'aide à leur organisation. Nous invitons toutes les sections locales intéressées à se rapprocher de la Commission Jeunes pour obtenir ce document et lancer de nouvelles RJP ! Les RJP de Grenoble ont d'ailleurs vu le jour en 2016 avec beaucoup de succès, et une deuxième édition vient de se tenir le 31 mars 2017.

\section{Le Congrès général}

Dans le cadre du prochain Congrès général de la SFP qui se tiendra à Orsay du 3 au 7 juillet 2017, la Commission Jeunes organise deux sessions : la première traitera de l'insertion professionnelle en dehors du monde de la recherche académique, avec notamment des témoignages de jeunes docteurs ainsi qu'une table ronde. La seconde session, préparée conjointement avec la Commission Enseignement, se concentrera sur la valorisation des compétences acquises lors du doctorat. Une soirée entre doctorants et postdoctorants est également prévue.

\section{Vulgarisation}

La Commission Jeunes a lancé une nouvelle action permettant aux jeunes physiciennes et physiciens (chercheurs-euses et étudiant-e-s) de publier des articles de vulgarisation portant sur leurs recherches, sur son nouveau site internet (http://jeunes.sfpnet.fr). Ces publications offriront une formidable opportunité pour donner la parole aux jeunes sur des sujets qui les passionnent, mais aussi pour montrer aux plus jeunes la diversité des parcours et des sujets abordés en physique. Les premiers articles traiteront par exemple des mécanismes offerts par le rein pour filtrer l'eau, de la superfluidité dans un mélange de bosons et de fermions, de télécommunications quantiques à base de nanotubes de carbone, et de dualité électromagnétique.

\section{Réseau des jeunes physicien-ne-s}

Un des rôles de la Commission Jeunes de la SFP est aussi d'apporter des conseils quant à l'orientation des étudiants (masters, stages, thèses, etc.), mais aussi à I'insertion professionnelle des jeunes docteurs (postdocs, débouchés, témoignages, etc.). Dans cette optique, la commission est en train de mettre en place une plateforme en ligne - sur son site - permettant aux jeunes physiciennes et physiciens de rejoindre un réseau pour répondre aux éventuelles questions d'autres jeunes.

\section{Beaucoup d'idées !}

D'autres actions sont en train de naitre au sein de la commission. Citons par exemple : des visites de laboratoires et de grands instruments pour les étudiants de licence et master (en collaboration avec les sections et divisions de la SFP), des échanges avec nos confrères allemands (jDPG) et italiens (AI-SF), notamment autour $d^{\prime}$ ateliers transfrontaliers ou d'excursions conjointes (visite du CERN, par exemple). 


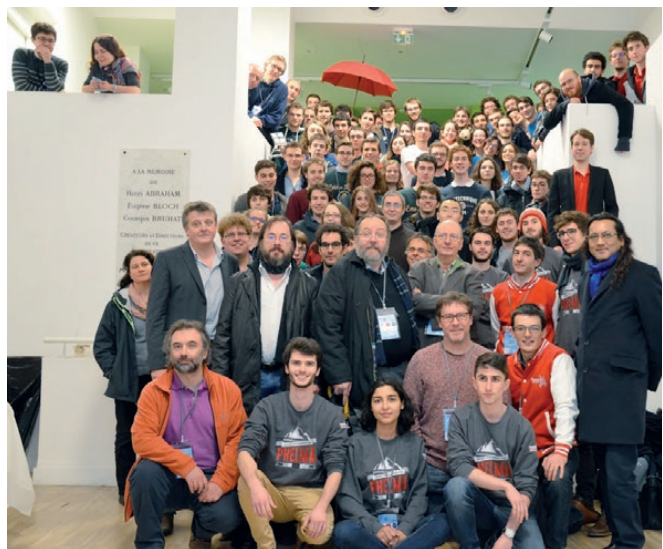

Les participants du French Physicists' Tournament 2017 (ENS Paris, 3-4 février 2017).

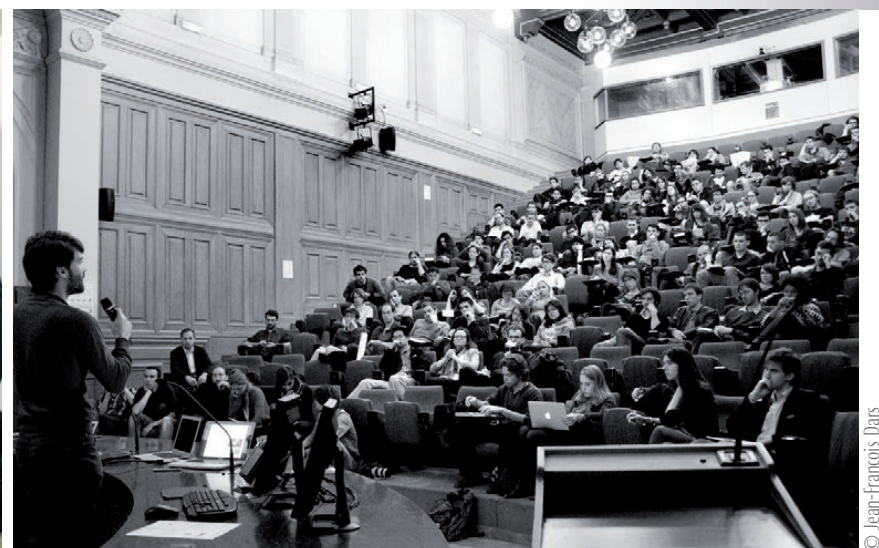

Les Rencontres des Jeunes Physiciennes (CNAM, Paris, 4 novembre 2014).

\section{Les événements qui concernent les jeunes}

Bien sûr, d'autres actions existent pour les jeunes, organisées par des divisions, sections ou commissions transverses. Citons ici trois exemples marquants.

\section{Les Olympiades de Physique}

Les Olympiades de Physique sont elles aussi une formation à la recherche destinée, cette fois, aux élèves des classes terminales. C'est un concours scientifique sur un projet à caractère expérimental, qui s'adresse à des équipes de lycéens encadrées par un professeur. Le concours est reconnu par le ministère de l'Éducation nationale. Créées en 1991 par I'Union des Professeurs de Physique et Chimie et la Société Française de Physique qui continuent d'en être les organisateurs, les Olympiades de Physique France ont joué un rôle de pionnier dans l'émergence des concours scientifiques sur projet destinés aux lycéens.

\section{Les Rencontres Physique-Entreprise- Recherche (RPER)}

Les premières Rencontres Physique-Entreprise-Recherche organisées par la SFP, regroupant une soixantaine d'entreprises et 420 doctorants et postdoctorants venus de toute la France, ont eu lieu à l'hôtel de ville de Paris le 10 mars 2017. Elles ont permis de mieux faire connaitre et valoriser la recherche, notamment la physique, faite dans les entreprises auprès des jeunes chercheuses et chercheurs, de faire apprécier tout le chemin - parfois tortueux - allant de la recherche fondamentale à l'innovation et de montrer combien l'esprit de recherche et la formation à la recherche sont aujourd'hui nécessaires aux entreprises.

Les journées de rencontre des jeunes chercheurs Les journées de rencontre des jeunes chercheurs (JRJC) ont été créées en 1991 par la division « Champs et particules » de la SFP, la Société Française d'Astronomie et $d^{\prime}$ Astrophysique (SF2A) et la Société Belge de Physique (BPS). Désormais organisées par les divisions "Champs et particules » et "Physique nucléaire » de la SFP, ces journées rassemblent durant une semaine, tous les ans et dans un lieu toujours différent, plus d'une cinquantaine de doctorants et jeunes postdoctorants de toute la France, pour leur permettre de présenter leurs résultats et d'échanger avec d'autres jeunes passionnés dans un cadre convivial.

\section{Les prix Jeunes Chercheurs-euses}

Tous les ans, la SFP remet trois prix de thèse : les prix René Pellat (physique des plasmas), Saint-Gobain et Daniel Guinier. Ces prix récompensent de jeunes docteurs dont les thèses sont remarquables pour leurs qualités, l'originalité des résultats obtenus, et la clarté des présentations.

\section{De l'énergie à revendre !}

\section{Un nouvel esprit insufflé au séminaire} de la SFP et à son Conseil d'administration Récemment, le séminaire annuel de la SFP - qui réunit membres du Conseil d'administration (CA) et du bureau, responsables des divisions, commissions et sections a connu un format inhabituel sous l'impulsion des jeunes. Le but était de sortir de ce séminaire avec une stratégie, des objectifs et des actions - ces dernières étant déclinées de manière collégiale et concrète. Le processus, mis en place par les jeunes, avait pour ambition de provoquer une effervescence intellectuelle collective dans une atmosphère décontractée et stimulante, de manière à faire émerger des idées, puis en débattre pour en dégager des actions à mener. Préparé par un CA spécial en juin 2016, ce séminaire s'est déroulé sur une journée et demie en décembre 2016 et a abouti à un document finalisé au CA de mars 2017, qui sera relayé auprès de tous dans notre revue Reflets de la Physique.

\section{Avoir confiance}

Les jeunes chercheuses et chercheurs représentent une source importante d'énergie et d'idées sur laquelle la SFP peut s'appuyer. Il ne faut donc pas hésiter à leur donner des responsabilités dans les différentes instances de la Société (sections, divisions, commissions), et y nommer un contact chargé de relayer et d'apporter un soutien aux actions menées par la Commission Jeunes. Essayer de lancer des Rencontres des Jeunes Physicie ${ }^{n} n^{e_{s}}$ dans de nouvelles régions peut, par exemple, être une réelle opportunité pour fédérer et cibler les forces vives qui pourront s'impliquer et s'épanouir au sein de la SFP.

Maxime Harazi Président de la Commission Jeunes (jeunes@sfpnet.fr) Michel Spiro Président de la Société Française de Physique 